\title{
Joint Scheduling and Resource Allocation in Uplink OFDM Systems for Broadband Wireless Access Networks
}

\author{
Jianwei Huang, Vijay G. Subramanian, Rajeev Agrawal, and Randall Berry
}

\begin{abstract}
Orthogonal Frequency Division Multiplexing (OFDM) with dynamic scheduling and resource allocation is a key component of most emerging broadband wireless access networks such as WiMAX and LTE (Long Term Evolution) for 3GPP. However, scheduling and resource allocation in an OFDM system is complicated, especially in the uplink due to two reasons: (i) the discrete nature of subchannel assignments, and (ii) the heterogeneity of the users' subchannel conditions, individual resource constraints and application requirements. We approach this problem using a gradient-based scheduling framework. Physical layer resources (bandwidth and power) are allocated to maximize the projection onto the gradient of a total system utility function which models application-layer Quality of Service (QoS). This is formulated as a convex optimization problem and solved using a dual decomposition approach. This optimal solution has prohibitively high computational complexity but reveals guiding principles that we use to generate lower complexity sub-optimal algorithms. We analyze the complexity and compare the performance of these algorithms via extensive simulations.
\end{abstract}

Index Terms-Orthogonal Frequency Division Multiplexing (OFDM), scheduling, resource allocation, optimization, dual decomposition, uplink communications

\section{INTRODUCTION}

$\mathbf{O}$ RTHOGONAL Frequency Division Multiplexing (OFDM) is the core technology for most recent wireless data systems, including IEEE 802.16 (WiMAX), IEEE 802.11a/g (Wireless LANs), and LTE for 3GPP. In this paper, we analyze an uplink scheduling and resource

Manuscript received 15 January 2008; revised 15 August 2008. Part of this work was done while J. Huang and V. G. Subramanian were at Motorola. J. Huang is supported by the Competitive Earmarked Research Grants (Project Number 412308) established under the University Grant Committee of the Hong Kong Special Administrative Region, China, the Direct Grant (Project Number C001-2050398) of The Chinese University of Hong Kong, and the National Key Technology R\&D Program (Project Number 2007BAH17B04) established by the Ministry of Science and Technology of the People's Republic of China.

V. Subramanian is supported by SFI grant 03/IN3/I346.

R. Berry was supported in part by the Motorola-Northwestern Center for Seamless Communications and NSF CAREER award CCR-0238382. The work was partially presented at the 2007 Asilomar Conference on Signals, Systems and Computers.

J. Huang is with the Department of Information Engineering, The Chinese University of Hong Kong (e-mail: jwhuang@ie.cuhk.edu.hk).

V. G. Subramanian is with the Hamilton Institute, National University of Ireland (e-mail: vijay.subramanian@nuim.ie).

R. Agrawal is with the Advanced Networks and Performance Dept., Motorola Inc., (e-mail: Rajeev.Agrawal@motorola.com).

R. Berry is with the Dept. of EECS, Northwestern University (e-mail: rberry@ece.northwestern.edu).

Digital Object Identifier 10.1109/JSAC.2009.090213. allocation problem for OFDM wireless access networks. The specific problem is motivated by the WiMAX/802.16e standard, where there is a centralized scheduler that knows the QoS classes, and can estimate the queue-lengths on each mobile device. The WiMAX/802.16e standard specifies mechanisms for communicating this information to the scheduler and for conveying the scheduling decisions to the mobiles, both with low delays. ${ }^{1}$

Our approach is motivated by our previous work on downlink scheduling in CDMA systems [3] and OFDM systems [4]. As in [3], [4], we consider a gradient-based scheduling framework, which is described in detail in Section II along with our system model. In this framework, the time-varying gradient of a utility function is used to guide the resource allocation decisions and provide long-term Quality of Service (QoS) guarantees. In particular, we maximize a weighted sumrate during each scheduling interval, where the weights are time-varying. The optimization variables are the assignment of OFDM subchannels to the users and the allocation of each user's power across the assigned subchannels. We highlight two challenging aspects of this problem in the OFDM uplink context. First, the discrete nature of subchannel assignments in OFDM systems usually leads to difficult integer programming problems. Second, the per-user power constraints in the uplink make the problem even less tractable. We initially relax the integer constraints and allow multiple users to share one subchannel using orthogonalization (e.g. via timesharing $^{2}$ ). In Section III we derive an optimal solution to this relaxed problem via a dual decomposition. Due to the peruser power constraints, the resulting algorithm has very high computational complexity. However, this provides insights into the structure of an optimal solution. In Section V we use the insights gained from the optimal solution to propose a family of sub-optimal algorithms that also take into account the integer constraints on subchannel allocations. Finally, in Section VI we these algorithms using simulation.

Most initial work on OFDM scheduling and resource allocation focused on the downlink case. The optimality conditions and algorithms derived for the downlink, however, can not be directly applied to the uplink due to differences in the resource constraints (see Section IV for a detailed discussion).

\footnotetext{
${ }^{1}$ Our model is also appropriate for LTE [29] for 3GPP, UMB [30] for 3GPP2 and the FLASH OFDM system [19] from Qualcomm Flarion.

${ }^{2}$ While super-position coding would yield an even larger (and more tractable) capacity region, we do not consider it as it is still not practical.
} 
Recently, uplink OFDM resource allocation has received some attention, including [21]-[28]. In [21], an iterative OFDM resource allocation was proposed to find a Nash Bargaining solution. A heuristic algorithm that tries to minimize each user's transmission power while satisfying the individual rate constraints is given in [22]. In [23], an algorithm for maximizing the sum-rate assuming Rayleigh fading is given; this is a special case of the problem considered here with equal weights. In our case no assumption on the fading distribution is made. In [24], an uplink problem with multiple antennas at the base station is considered; here, we focus on single antenna systems.

The work in [25]-[28] is closer to ours. In [25], a weighted rate maximization problem is also considered for the uplink, but with static weights. The results in [25] are generalized in [26] to account for per-time slot fairness via a utility function of the instantaneous rate. Per time-slot fairness is also considered in [27]. Our work differs from these in that by using a gradient-based scheduler, we can consider longterm fairness, which depends on the average rate or queue sizes. For elastic data applications, long-term QoS evaluation is more reasonable than the short-term QoS evaluation during each time slot. It not only more faithfully reflects users' actual perceived performance, but also gives the system more flexibility in terms of exploiting multi-user diversity. Finally, [28] proposed a heuristic algorithm based on Lagrangian relaxation, which has high complexity due to a subgradient search of the dual variables. Here we use Lagrangian relaxation to give an optimal solution of the uplink problem when time-sharing is allowed. Solving this problem provides both an upperbound on the actual system performance as well as the intuition we use to design good heuristic algorithms.

\section{Problem Statement}

We consider the problem of scheduling and resource allocation for the uplink of a OFDM cell, where a set $\mathcal{M}=$ $\{1, \ldots, M\}$ of users transmit to the same base station. The total frequency band is divided into a set $\mathcal{N}=\{1, \ldots, N\}$ of subchannels (e.g., frequency bands). Let $p_{i j}$ be the power user $i$ allocates to subchannel $j$, which is subject to a per-user power constraint:

$$
\sum_{j} p_{i j} \leq P_{i}, \forall i \in \mathcal{M}
$$

Let $x_{i j}$ be the fraction of subchannel $j$ allocated to user $i$, where the total allocation across all users should be no larger than 1 , i.e.,

$$
\sum_{i} x_{i j} \leq 1, \forall j \in \mathcal{N}
$$

We use bold symbols to denote vectors and matrices of these quantities, e.g., $\boldsymbol{P}=\left\{P_{i}, \forall i\right\}, \boldsymbol{e}=\left\{e_{i j}, \forall i, j\right\}, \boldsymbol{p}=$ $\left\{p_{i j}, \forall i, j\right\}$, and $\boldsymbol{x}=\left\{x_{i j}, \forall i, j\right\}$.

Time is divided into equal length slots. At the beginning of every time slot, the scheduler seeks to maximize a (timevarying) weighted sum of the users' rates over a (time-varying) rate-region. We describe this rate-region next.

The scheduler is assumed to have knowledge of the normalized received signal-to-interference plus noise ratio (SINR) per unit transmit power, $e_{i j}$, for each user $i$ and subchannel $j .^{3}$ The time-varying subchannel quality vector at time $t$ is denoted by $\boldsymbol{e}_{t}$. As in [4], this model incorporates various subchannelization schemes where the resource allocation is performed in terms of subchannels (i.e., sets of tones); $e_{i j}$ represents a collective quality indicator for the subchannel, e.g., the (harmonic/geometric/arithmetic) average across the tones in the subchannel. This model also applies if resource allocation is done with a granularity of multiple symbols in the time domain.

We model the feasible rate region at time $t$ by

$$
\mathcal{R}\left(\boldsymbol{e}_{t}\right)=\left\{\boldsymbol{r}: r_{i}=\sum_{j \in \mathcal{N}} f\left(x_{i j}, p_{i j} e_{i j}(t)\right)\right\},
$$

where $(\boldsymbol{x}, \boldsymbol{p}) \in \mathcal{X}$ are chosen subject to (1) and (2) and the set

$$
\mathcal{X}:=\left\{(\boldsymbol{x}, \boldsymbol{p}) \geq \mathbf{0}: 0 \leq x_{i j} \leq 1, p_{i j} \leq \frac{x_{i j} s_{i j}}{e_{i j}(t)} \forall i, j\right\} .
$$

Here, $f(a, b)=a \log \left(1+\frac{b}{a}\right)$ so that $r_{i}$ is the achievable rate of user $i$ in a Gaussian Multiple Access Channel using timesharing (cf. [17, Section 15.3.6, pg. 547]). By continuity, we assume that $f(0, b)=0$. The value of $s_{i j}$ is a maximum SINR constraint on subchannel $j$ for user $i$, which can model scenarios when users have limited choices of modulation and coding schemes.

In practical OFDM systems, $x_{i j}$ is constrained to be an integer, i.e., we have the additional constraint $x_{i j} \in\{0,1\}$ for all $i, j$. Initially, we ignore this constraint, and consider a system in which users can share each tone. If resource allocation is done on blocks of OFDM symbols, then fractional values of $x_{i j}$ can be implemented by time-sharing the symbols in a block. Alternatively, this can also be implemented by frequency sharing (e.g., [31]), if there are a large number of subchannels with roughly equal gains. We will re-introduce these integer constraints in Sections IV and V.

Next we formulate the scheduling and resource allocation problem. Our approach is based on the gradient-based scheduling framework in [2], [10], [12]. Each user $i$ is assigned a utility function $U_{i}\left(W_{i, t}, Q_{i, t}\right)$ depending on their average throughput $W_{i, t}$ up to time $t$ and their queue-length $Q_{i, t}$ at time $t$. This is used to quantify fairness and ensure stability of the queues. At the beginning of each time slot $t$, the scheduler chooses a $\boldsymbol{r}_{t} \in \mathcal{R}\left(\boldsymbol{e}_{t}\right)$ that maximizes a weighted sum of the users' rates, where the weights are determined by the gradient of the sum utility across all users, i.e., it solves

$$
\max _{\boldsymbol{r}_{t} \in \mathcal{R}\left(\boldsymbol{e}_{t}\right)}\left(\nabla_{\boldsymbol{w}} U\left(\boldsymbol{W}_{t}, \boldsymbol{Q}_{t}\right)-\nabla_{\boldsymbol{q}} U\left(\boldsymbol{W}_{t}, \boldsymbol{Q}_{t}\right)\right)^{T} \boldsymbol{r}_{t},
$$

where $U\left(\boldsymbol{W}_{t}, \boldsymbol{Q}_{t}\right)=\sum_{i=1}^{K} U_{i}\left(W_{i, t}, Q_{i, t}\right)$. Further assuming that for each user $i, U_{i}\left(W_{i, t}, Q_{i, t}\right)=u_{i}\left(W_{i, t}\right)-\frac{d_{i}}{p}\left(Q_{i, t}\right)^{p}$, then (5) is equivalent to

$$
\max _{\boldsymbol{r}_{t} \in \mathcal{R}\left(\boldsymbol{e}_{t}\right)} \sum_{i}\left(\frac{\partial u_{i}\left(W_{i, t}\right)}{\partial W_{i, t}}+d_{i}\left(Q_{i, t}\right)^{p-1}\right) r_{i, t},
$$

${ }^{3}$ In both FDD and TDD systems this can be obtained using a combination of measurements made on the uplink pilots as well as past transmissions from the mobiles. 
where, $u_{i}\left(W_{i, t}\right)$ is a increasing concave function used to represent elastic data applications (e.g., [1], [8], [13], [18]), $d_{i} \geq 0$ is a QoS weight for user $i$ 's queue length, and $p>1$ is a fairness parameter associated with the queue length.

The broad class of policies in (6) can be tuned to yield good operating points by a proper choice of parameters. If $d_{i}=0$ for all $i \in \mathcal{M}$, the resulting policy has been shown to yield utility maximizing solutions (see [2], [10], [12]). If $u_{i}(\cdot) \equiv 0$ with $d_{i}>0$ for all $i \in \mathcal{M}$, then the policy has been shown to be stabilizing in a variety of settings (see [5][7]). The weights can also be adapted so as to maximize sum utility subject to stability [9] or (feasible) minimum throughput constraints (see [11]).

More generally, the optimization in (6) can be written as

$$
\max _{\boldsymbol{r}_{t} \in \mathcal{R}\left(\boldsymbol{e}_{t}\right)} \sum_{i} w_{i, t} r_{i, t},
$$

where $w_{i, t} \geq 0$ is a time-varying weight assigned to the $i$ th user at time $t$. Our focus is on solving such a problem for an uplink OFDM system, i.e., when $\mathcal{R}\left(\boldsymbol{e}_{t}\right)$ is given by (3). Note that (7) must be re-solved at each scheduling instant because of changes in both the subchannel state, $e_{t}$, and the weights. While in the above examples, the weight $w_{i, t}$ is given by the gradient of an utility function, our algorithms also apply to other methods for generating these weights.

\section{Optimal SOLUTiOn With Fractional Allocations}

We now consider the optimal solution to (7) when $\mathcal{R}\left(\boldsymbol{e}_{t}\right)$ is given by (3). Suppressing the time index, the problem is

$$
\max _{(\boldsymbol{x}, \boldsymbol{p}) \in \mathcal{X}} \sum_{i \in \mathcal{M}} w_{i} \sum_{j \in \mathcal{N}} x_{i j} \log \left(1+\frac{p_{i j} e_{i j}}{x_{i j}}\right)
$$

subject to the per subchannel assignment constraints in (2) and the per user power constraints in (1), where $\mathcal{X}$ is given in (4).

It can be shown that Problem UL has no duality gap and so we solve it via a dual formulation. We associate dual variables $\boldsymbol{\lambda}=\left(\lambda_{i}\right)_{i \in \mathcal{M}}$ with constraints $(1)$ and $\boldsymbol{\mu}=\left(\mu_{j}\right)_{j \in \mathcal{N}}$ with constraints $(2)$, resulting in the Lagrangian,

$$
\begin{aligned}
& L(\boldsymbol{\lambda}, \boldsymbol{\mu}, \boldsymbol{x}, \boldsymbol{p}):=\sum_{i, j} w_{i} x_{i j} \log \left(1+\frac{p_{i j} e_{i j}}{x_{i j}}\right) \\
&+\sum_{i} \lambda_{i}\left(P_{i}-\sum_{j} p_{i j}\right)+\sum_{j} \mu_{j}\left(1-\sum_{i} x_{i j}\right)
\end{aligned}
$$

Therefore, the optimal solution to Problem UL is given by

$$
\min _{(\boldsymbol{\lambda}, \boldsymbol{\mu}) \geq \mathbf{0}} \max _{(\boldsymbol{x}, \boldsymbol{p}) \in \mathcal{X}} L(\boldsymbol{\lambda}, \boldsymbol{\mu}, \boldsymbol{x}, \boldsymbol{p}) .
$$

We solve this problem by the following steps. First, we analytically find the optimal $\boldsymbol{p}$ and $\boldsymbol{x}$ given fixed values of the dual variables. We then show that the optimal $\boldsymbol{\mu}$ is given by a search for the maximum value of a per-user metric on each subchannel. The final step is to numerically search for the optimal value of $\lambda$.

The value of $\boldsymbol{p}$ which maximizes $L(\boldsymbol{\lambda}, \boldsymbol{\mu}, \boldsymbol{x}, \boldsymbol{p})$ given $\boldsymbol{x}, \boldsymbol{\mu}$ and $\boldsymbol{\lambda}$ is given bu

$$
p_{i j}^{*}=\frac{x_{i j}}{e_{i j}} \min \left\{\left(\frac{w_{i} e_{i j}}{\lambda_{i}}-1\right)^{+}, s_{i j}\right\},
$$

where $(\cdot)^{+}=\max (\cdot, 0)$. Substituting $\boldsymbol{p}^{*}$ into $L(\cdot, \cdot, \cdot, \cdot)$ yields

$$
\begin{aligned}
L(\boldsymbol{\lambda}, \boldsymbol{\mu}, \boldsymbol{x})=\sum_{i j} x_{i j}\left(w_{i} h\left(\lambda_{i}, w_{i} e_{i j}, s_{i j}\right)-\mu_{j}\right) & \\
& +\sum_{j} \mu_{j}+\sum_{i} \lambda_{i} P_{i}
\end{aligned}
$$

where we have used the function $h(\cdot, \cdot, \cdot)$ from [3]; namely,

$$
h(a, b, c)= \begin{cases}0 & \text { if } a \geq b \\ \frac{a}{b}-1-\log \frac{a}{b} & \text { if } \frac{b}{1+c} \leq a<b \\ \log (1+c)-\frac{a}{b} c & \text { if } a<\frac{b}{1+c},\end{cases}
$$

where $a \geq 0, b>0$ and $c \geq 0$. Optimizing (11) over $\boldsymbol{x}$ such that $x_{i j} \in[0,1]$ yields

$$
\begin{aligned}
L(\boldsymbol{\lambda}, \boldsymbol{\mu})=\sum_{j} \mu_{j} & +\sum_{i} \lambda_{i} P_{i} \\
& +\sum_{i j}\left(w_{i} h\left(\lambda_{i}, w_{i} e_{i j}, s_{i j}\right)-\mu_{j}\right)^{+},
\end{aligned}
$$

where the optimal subchannel allocation has the following structure

$$
x_{i j}^{*}\left(\mu_{j}\right)= \begin{cases}1, & \text { if } w_{i} h\left(\lambda_{i}, w_{i} e_{i j}, s_{i j}\right)>\mu_{j} \\ {[0,1],} & \text { if } w_{i} h\left(\lambda_{i}, w_{i} e_{i j}, s_{i j}\right)=\mu_{j} \\ 0, & \text { if } w_{i} h\left(\lambda_{i}, w_{i} e_{i j}, s_{i j}\right)<\mu_{j} .\end{cases}
$$

Since the cost function in (13) is separable, by defining $\mu_{i j}(\cdot):=w_{i} h\left(\cdot, w_{i} e_{i j}, s_{i j}\right)$ as in [3], we can minimize $L(\boldsymbol{\lambda}, \boldsymbol{\mu})$ over $\boldsymbol{\mu}$ for a given $\boldsymbol{\lambda}$ by setting $\mu_{j}=\mu_{j}^{*}(\boldsymbol{\lambda})$ given by

$$
\mu_{j}^{*}(\boldsymbol{\lambda})=\max _{i} \mu_{i j}\left(\lambda_{i}\right)
$$

From (14) and (15), it is clear that $x_{i j}^{*}\left(\mu_{j}^{*}(\boldsymbol{\lambda})\right) \equiv 0$ if $i \notin \arg \max _{i \in \mathcal{M}} \mu_{i j}\left(\lambda_{i}\right)$, i.e., users not maximizing a specific subchannel metric are not allocated the subchannel. There will be ties when multiple users achieve the value $\mu_{j}^{*}$ on subchannel $j$. These can be broken arbitrarily for optimizing the dual function. Substituting $\boldsymbol{\mu}^{*}$ into $L(\boldsymbol{\lambda}, \boldsymbol{\mu})$, and noticing that $\boldsymbol{\mu}^{*}$, $\boldsymbol{x}^{*}, \boldsymbol{p}^{*}$ are all functions of $\boldsymbol{\lambda}$, we have

$$
L(\boldsymbol{\lambda}):=\sum_{j} \max _{i} \mu_{i j}\left(\lambda_{i}\right)+\sum_{i} \lambda_{i} P_{i} .
$$

The solution to (9) is given by numerically minimizing $L(\boldsymbol{\lambda})$ over $\boldsymbol{\lambda} \geq \mathbf{0}$. For this we use a subgradient-based search and update $\lambda$ by

$$
\lambda_{i}(t+1)=\left[\lambda_{i}(t)-\kappa(t)\left(P_{i}-\sum_{j} p_{i j}^{*}(t)\right)\right]^{+}, \forall i \in \mathcal{M} .
$$

where $p_{i j}^{*}$ is given by (10) and $x_{i j}$ are given by (14) and additionally satisfy the feasibility constraint (2) in case of ties. The algorithm will converge when $\kappa(t)$ is chosen appropriately, e.g., [20, Exercise 6.3.2]. Given an optimal $\lambda^{*}$, by duality, $L\left(\boldsymbol{\lambda}^{*}\right)$ is the optimal objective value to Problem UL.

However, to implement this algorithm, the scheduler must specify the corresponding optimal primal values of $\left(\boldsymbol{x}^{*}, \boldsymbol{p}^{*}\right)$. Here, as in [4], more care is required. Specifically, when ties occur in (15), it is often needed to split the subchannel among several users (i.e., allowing fractional values of $\boldsymbol{x}^{*}$ ). Following a similar approach as in [3] (for the downlink), the optimal 
fractional values can be found by solving a linear program whose size increases with the number of users and tones involved in each tie. As discussed below, this number can be quite large in the uplink setting. Moreover, as noted earlier, one is typically interested in an integer allocation in practice. We consider this problem next.

\section{Integer Subchannel Allocation BASEd on OPTIMAL AlgORITHM}

We now address the problem:

$$
\max _{\substack{(\boldsymbol{x}, \boldsymbol{p}) \in \mathcal{X}, x_{i j} \in\{0,1\}, \forall i, j}} \sum_{i \in \mathcal{M}} w_{i} \sum_{j \in \mathcal{N}} x_{i j} \log \left(1+\frac{p_{i j} e_{i j}}{x_{i j}}\right),
$$

subject to per user power constraints (1). Initially, consider the following heuristic for Problem UL-Int: (i) Solve Problem UL as in the previous section; and (ii) "break" any ties on all subchannels, i.e., whenever there is a fractional $x_{i j}^{*}$ value, choose one user in the tie and allocate subchannel $j$ to that user only. Clearly, if there are no ties, this algorithm gives the optimal solution to Problem UL-Int. After all ties are broken, we can then re-optimize the power allocation for each user using a finite-time water-filling algorithm as in [4].

In [4] a similar procedure is used for a downlink OFDM scheduling problem. However, there are several major differences between the uplink and downlink settings that make this approach less appealing for the uplink. First, in the downlink case there is a single power constraint $\sum_{i, j} p_{i j} \leq P$ for the base station instead of the per-user constraints in (1). Hence, in the downlink $L(\lambda)$ is a function of only a single dual variable $\lambda$, which simplifies the numerical search for the optimizer. In the uplink setting, the convergence of the subgradient search is too slow to be useful for scheduling on a fast time-scale.

Second, even if the optimal $\boldsymbol{\lambda}$ can be found, breaking ties is more difficult than in the downlink case. Scalar subgradients of $L(\lambda)$ in the downlink case can be used to devise simple tie-breaking rules [4], while in the uplink case, the subgradients are vectors, complicating such an approach. Also, the uplink case can be more sensitive to how ties are resolved. For example, if two users, $i$ and $l$, have the same weights $\left(w_{i}=w_{l}\right)$ and the same gains on subchannel $j\left(e_{i j}=e_{l j}\right)$, then allocating subchannel $j$ to either yields the same total weighted rate and the same total power usage in the downlink case. On the other hand, different allocations lead to different individual power consumptions in the uplink case, and thus may lead to totally different solutions.

Finally, the number of ties is typically much larger in the uplink case than in the downlink case. Consider a simple scenario with two users and two subchannels. Each user has the same gain over both subchannels, i.e., $e_{i 1}=e_{i 2}=e_{i}$ for $i=1,2$, and $P=P_{1}=P_{2}$, where $P$ is the total power constraint in the downlink case. Assume user 2 has a much better subchannel than user 1 so that in the downlink case, the unique optimal solution is to allocate both subchannels to user 2, and there is no tie. However, in the uplink case, it can be shown that at the optimal dual solution, $\lambda_{1}$ and $\lambda_{2}$ will satisfy $\mu_{1 j}\left(\lambda_{1}\right)=\mu_{2 j}\left(\lambda_{2}\right)$ for $j=1$ and 2 , i.e., there is a tie in each subchannel and four possible subchannel allocations must be considered to determine how to break the tie. This can be easily extended to $M$ users and $N$ subchannels, with each user having the same gain over all its subchannels, resulting in $M^{N}$ ties even in this simplistic setting.

\section{Low Complexity Suboptimal Algorithms}

Due to the issues discussed in the previous section, finding the optimal dual solution to Problem UL and breaking any ties to get an integer allocation is computationally difficult, even for a moderately sized system. Thus, we now present a family of sub-optimal algorithms (SOAs), which try to reduce this complexity using the structure revealed by the optimal algorithm while enforcing an integer-tone allocation and exhibiting good performance.

In the optimal algorithm, given the optimal $\lambda^{*}$, the optimal subchannel allocation up to any ties is determined by sorting the users on each tone according to the metric $\mu_{i j}\left(\boldsymbol{\lambda}^{*}\right)$ as in (14). Given an optimal subchannel allocation, the optimal power allocation is given by a per-user water-filling allocation as in (10). In each SOA, we use the same two phases but modify them to reduce the complexity. Specifically, we begin with a subChannel Allocation (CA) phase which assigns each subchannel to at most one user. Two different implementations of the CA phase are given. In SOA1, instead of using $\mu_{i j}\left(\boldsymbol{\lambda}^{*}\right)$, we consider metrics based on a constant power allocation over all subchannels assigned to a user. In SOA2, we again use a dual based approach, but here we first determine the number of subchannels assigned to each user and then match specific subchannels and users. After the CA phase in both SOAs, we execute the Power Allocation (PA) phase in which each user's power is allocated across the assigned subchannels as in the optimal algorithm.

\section{A. CA in SOA1: Progressive Subchannel Allocation based on Metric Sorting}

In this family of SOAs, subchannels are assigned sequentially in one pass based on a per user metric for each subchannel. Let $\mathcal{K}_{i}(n)$ denote the set of subchannels assigned to user $i$ after the $n$th iteration. Let $g_{i}(n)$ denote user $i$ 's metric during the $n$th iteration and let $l_{i}(n)$ be the subchannel index that user $i$ would like to be assigned if he/she is assigned the $n$th subchannel. The resulting CA algorithm is given in Algorithm 1. Note that all the user metrics are updated after each subchannel is assigned.

\begin{tabular}{l}
\hline Algorithm 1 CA Phase for SOA1 \\
\hline 1: Initialization: set $n=0$ and $\mathcal{K}_{i}(n)=\emptyset$ for each user $i$. \\
2: while $n<N$ do \\
3: $\quad n=n+1$. \\
4: $\quad$ Update subchannel index $l_{i}(n)$ for each user $i$. \\
5: $\quad$ Update metric $g_{i}(n)$ for each user $i$. \\
6: $\quad$ Find $i^{*}(n)=$ arg $\max _{i} g_{i}(n)$ (break ties arbitrarily). \\
7: $\quad$ Assign the $n$th subchannel to user $i^{*}(n):$
\end{tabular}


We consider several variations of Algorithm 1 which correspond to different choices for steps 4 and 5 . The choices for step 4 are:

(4A): Sort the subchannels based on the best channel condition among all users. This involves two steps. First, for each subchannel $j$, find the best channel condition among all users and denote it by $\tilde{\mu}_{j}:=\max _{i} e_{i j}$. Second, find a subchannel permutation $\left\{\alpha_{j}\right\}_{j \in \mathcal{N}}$ such that $\tilde{\mu}_{\alpha_{1}} \geq \tilde{\mu}_{\alpha_{2}} \geq \cdots \geq \tilde{\mu}_{\alpha_{N}}$, and set $l_{i}(n)=\alpha_{n}$ for each user $i$ at the $n$th iteration. Each $\max$ operation has complexity of $O(M)$, and the sorting operation has a complexity of $O(N \log (N))$. The total complexity is $O(N M+N \log N)$. We note that this is a one-time "preprocessing" that needs to done before the CA phase starts. During the subchannel allocation iterations, the users just choose the subchannel index from the sorted list.

$(4 B)$ : Sort the subchannels based on the channel conditions for each individual user. For each user $i$ at the $n$th iteration, set $l_{i}(n)$ to be the subchannel index with the largest gain among all unassigned subchannels, i.e., $l_{i}(n)=$ $\arg \max _{j \in \mathcal{N} \backslash \cup_{i} \mathcal{K}_{i}(n-1)} e_{i j}$. This requires $M$ sorts (one per user) that only need to be performed once, since each subchannel assignment does not change a user's ordering of the remaining subchannels. The total complexity of the $M$ sorting operations is $O(M N \log N)$, which is higher than that in (4A).

Let $k_{i}(n)=\left|\mathcal{K}_{i}(n)\right|$. The choices for step 5 are:

$(5 A)$ : Set $g_{i}(n)$ to be the total increase in user $i$ 's utility if assigned subchannel $l_{i}(n)$, assuming constant power allocation over all assigned subchannels, i.e.,

$$
\begin{array}{r}
g_{i}(n)=w_{i}\left[\sum_{j \in \mathcal{K}_{i}(n-1) \cup\left\{l_{i}(n)\right\}} \log \left(1+\frac{P_{i} e_{i j}}{k_{i}(n-1)+1}\right)\right. \\
\left.-\sum_{j \in \mathcal{K}_{i}(n-1)} \log \left(1+\frac{P_{i} e_{i j}}{k_{i}(n-1)}\right)\right] .
\end{array}
$$

$(5 B)$ : Set $g_{i}(n)$ to be user $i$ 's gain from only subchannel $l_{i}(n)$, assuming constant power allocation, i.e.,

$$
g_{i}(n)=w_{i} \log \left(1+\frac{P_{i}}{k_{i}(n-1)+1} e_{i, l_{i}(n)}\right) .
$$

Compared with $(5 A)$, this metric ignores the change in user $i$ 's utility due to the decrease in power allocated to any subchannels in $\mathcal{K}_{i}(n-1)$.

The complexity of either of these choices over $N$ iterations is $O(N M)$, and so the total complexity for the CA phase ${ }^{4}$ is $O(N M+N \log N)$ (if $(4 A)$ is chosen) or $O(M N \log N)$ (if $(4 B)$ is chosen).

B. CA in SOA2: subchannel Number Assignment \& subchannel User Matching

As summarized in Algorithm 2, SOA2 implements the CA phase through two steps: subchannel number assignment (CNA) and subchannel user matching (CUM).

CNA Step: The CNA step determines the number of subchannels $n_{i}$ assigned to each user $i \in \mathcal{M}$ based on the

\footnotetext{
${ }^{4}$ We note that SOA1 with $(4 B)$ and $(5 B)$ is similar to the algorithms proposed in [25]. In Section VI we show that other variations of SOA1 $((4 B)$ and $(5 A))$ and SOA2 can achieve better performance with similar or slightly higher complexity.
}

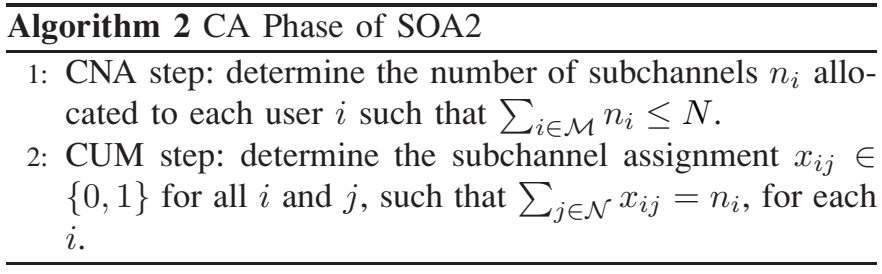

approximation that each user sees a flat wide-band fading subchannel. This step does not specify which subchannel is allocated to which user; such a mapping is left to the CUM step. The CNA step is further divided into two stages: a basic assignment stage and an assignment improvement stage.

Stage 1, Basic Assignment: Here, we model each user $i$ as having a normalized SINR $\bar{e}_{i}=\frac{1}{N} \sum_{j \in \mathcal{N}} e_{i j}$ over all subchannels, and then determine the number of subchannels assigned $n_{i}$ to user $i$ by solving:

$$
\begin{array}{ll}
\max _{\left\{n_{i} \geq 0, i \in \mathcal{M}\right\}} & \sum w_{i \in \mathcal{M}} n_{i} \log \left(1+\frac{P_{i}}{n_{i}} \bar{e}_{i}\right) \\
\text { subject to: } & \sum_{i \in \mathcal{M}} n_{i} \leq N .
\end{array}
$$

(SOA2-CNA)

It can be shown that Problem SOA2-CNA is a standard concave maximization problem over a convex set with a unique and possibly non-integer solution; we use a dual relaxation method to find this solution. The optimal Lagrange multiplier for the subchannel constraint and any intermediate optimal $n_{i}$ allocation can be found by a line-search, over ranges $\left[0, \max _{i}\left(w_{i} \log \left(1+P_{i} \bar{e}_{i}\right)\right)\right]$ and $[0, N]$, respectively. Hence, the worst case complexity of solving each subproblem with a fixed dual variable is independent of $M$ or $N$. Since we need to determine the value of $n_{i}$ for every user, the complexity of the basic assignment step is $O(M)$. If the resultant channel allocations contain non-integer values, we will approximate with an integer solution that satisfies $\sum_{i \in \mathcal{M}} n_{i}=N$.

Since each user is allocated only a subset of the subchannels, the normalized SINR $\bar{e}_{i}=\frac{1}{N} \sum_{j \in \mathcal{N}} e_{i j}$ is typically a pessimistic estimate of the averaged subchannel conditions over the allocated subset. This motivates the following assignment improvement stage of CNA.

Stage 2, Assignment Improvement: Here, we iteratively solve the following variation of Problem SOA2-CNA:

$$
\begin{aligned}
\max _{\left\{n_{i}(t) \geq 0, i \in \mathcal{M}\right\}} & \sum_{i \in \mathcal{M}} w_{i} n_{i}(t) \log \left(1+\frac{P_{i}}{n_{i}(t)} \bar{e}_{i}(t)\right) \\
\text { subject to: } & \sum_{i \in \mathcal{M}} n_{i}(t) \leq N, \quad \text { (SOA2-CNA-t) }
\end{aligned}
$$

for $t=1,2, \ldots$ During the $t$-th iteration, $\bar{e}_{i}(t)$ is a refined estimate of the normalized SINR based on the best $n_{i}(t-1)$ subchannels of user $i\left(n_{i}(0)=N\right)$. The iterative procedure stops when the subchannel allocation converges or the maximum number of iterations allowed is reached. At the end an integer approximation will be performed, if needed.

The complete algorithm for the CNA phase of SOA2 is given in Algorithm 3. In order to perform the assignment improvement, we need to perform $M$ sorting operations once, with a total complexity $O(M N \log (N))$. Step 4 of each iteration has complexity of $O(M)$ due to solving $M$ subproblems 
for a fixed dual variable. The maximum number of iterations is fixed and thus is independent of $N$ or $M$. The integer approximation stage (typically) requires a sorting with the complexity of $O(M \log (M))$. So the total complexity for the CNA phase of SOA2 is $O(M N \log (N)+M \log (M))$.

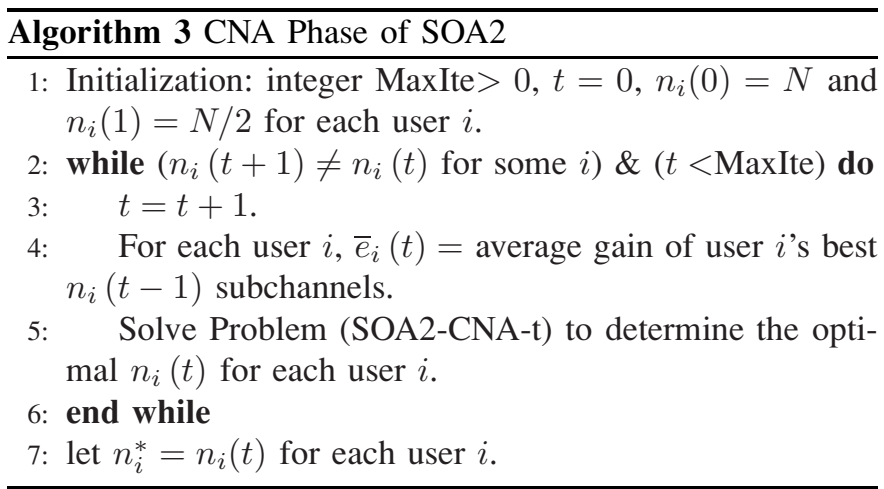

CUM Step: The CNA step determines how many subchannels are to be allocated to each user but not the exact subchannel assignment. This is refined in the CUM step by finding a subchannel assignment that maximizes the weightedsum rate assuming each user employs a flat power allocation, i.e., we solve the problem:

$$
\begin{aligned}
\max _{x_{i j} \in\{0,1\}} & \sum_{i \in \mathcal{M}} \sum_{j \in \mathcal{N}} x_{i j} w_{i} \log \left(1+\frac{P_{i}}{n_{i}^{*}} e_{i j}\right) \\
\text { subject to: } & \sum_{j \in \mathcal{N}} x_{i j}=n_{i}^{*}, \forall i \in \mathcal{M}, \quad \text { (SOA2-CUM) } \\
& \sum_{i \in \mathcal{M}} x_{i j}=1, \forall j \in \mathcal{N},
\end{aligned}
$$

where $\boldsymbol{n}^{*}=\left(n_{i}^{*}, i \in \mathcal{M}\right)$ is the integer subchannel allocation obtained in the CNA step.

Problem SOA2-CUM is an integer Assignment Problem whose optimal solution can be found by using the Hungarian Algorithm [15]. ${ }^{5}$ To use the Hungarian algorithm here, we need to perform the following "virtual user splitting": For user $i$, let $r_{i j}=w_{i} \log \left(1+\frac{P_{i}}{n_{i}^{*}} e_{i j}\right)$, and let

$$
\boldsymbol{r}_{i}=\left[r_{i 1}, r_{i 2}, \cdots, r_{i N}\right]
$$

be user $i$ 's achievable rates over all possible subchannels. We can then form an $M \times N$ matrix $\boldsymbol{R}=\left[\boldsymbol{r}_{1}^{T}, \boldsymbol{r}_{2}^{T}, \cdots, \boldsymbol{r}_{M}^{T}\right]^{T}$. Next, we split each user $i$ into $n_{i}^{*}$ virtual users by adding $n_{i}^{*}-1$ copies of the row vector $\boldsymbol{r}_{i}$ to the matrix $\boldsymbol{R}$ giving a $N \times N$ square matrix. Solving Problem SOA2-CUM is then equivalent to finding a permutation matrix $C^{*}=\left[c_{i j}\right]_{N \times N}$ such that

$$
\boldsymbol{C}^{*}=\arg \min _{\boldsymbol{C} \in \mathcal{C}}-\boldsymbol{C} \cdot \boldsymbol{R}:=\arg \min _{\boldsymbol{C} \in \mathcal{C}}-\sum_{i=1}^{N} \sum_{j=1}^{N} c_{i j} r_{i j} .
$$

This problem can be solved by the standard Hungarian algorithm which has a computational complexity of $O\left(N^{3}\right)$. After

\footnotetext{
${ }^{5} \mathrm{~A}$ similar idea has been used to solve other OFDM resource allocation problems, e.g., [16], [21].

${ }^{6}$ Here we assume that each user is allocated at least one subchannel. If only $\tilde{M}<M$ users are allocated positive amount of channels, we can replace $M$ by $\tilde{M}$ in the discussions.
}

TABLE I

Worst Case Computational Complexity of Suboptimal ALGORITHMS

\begin{tabular}{c|c|c|c}
\hline \hline \multicolumn{2}{c|}{ Suboptimal Algorithms } & Worst Case Complexity \\
\hline \hline \multirow{4}{*}{ SOA1 } & \multirow{3}{*}{$\begin{array}{c}\text { subChannel } \\
\text { Allocation }\end{array}$} & 4A 5A & $O(N M+N \log N)$ \\
\cline { 3 - 4 } & & 4A 5B & $O(N M+N \log N)$ \\
\cline { 2 - 4 } & 4B 5A & $O(M N \log N)$ \\
\cline { 2 - 4 } & \multicolumn{2}{|c|}{ Power Allocation } & $O(M N \log N)$ \\
\cline { 2 - 4 } & \multicolumn{2}{|c|}{ Total } & $O(M N)$ \\
\hline \multirow{4}{*}{ SOA2 } & SubChannel & CNA & $O(M N \log N+M \log M)$ \\
\cline { 2 - 4 } & Allocation & CUM & $\left.O(N)^{3}\right)$ \\
\cline { 2 - 4 } & \multicolumn{2}{|c|}{ Power Allocation } & $O(M N)$ \\
\cline { 2 - 4 } & \multicolumn{2}{|c|}{ Total } & $O\left(N^{3}+M N \log N+M \log M\right)$ \\
\hline
\end{tabular}

obtaining $C^{*}$, we can calculate the corresponding subchannel allocation $\boldsymbol{x}^{*}$, e.g., if $c_{k j}^{*}=1$ and virtual user $k$ corresponds to the actual user $i$, then $x_{i j}^{*}=1$.

\section{Power Allocation (PA) phase with Single-user Water-filling}

In this phase each user optimally allocates its power across the subchannel assigned to it in the CA phase. For user $i$, this can be formulated as the following problem

$$
\max _{\boldsymbol{p}_{i} \in \mathcal{P}_{i}} \sum_{j} x_{i j}^{*} \log \left(1+p_{i j} e_{i j}\right),
$$

where $\mathcal{P}_{i}=\left\{\boldsymbol{p}_{i} \geq 0: p_{i j} \leq \frac{s_{i j}}{e_{i j}}, \sum_{j \in \mathcal{N}} p_{i j} \leq P_{i}\right\}$.

The solution to Problem $\mathrm{PA}_{i}$ will be a water-filling type of power allocation, taking into account the per subchannel SINR constraint. Specifically, if $\sum_{j \in \mathcal{N}} x_{i j}^{*} \frac{s_{i j}}{e_{i j}} \leq P_{i}$, we let $p_{i j}^{*}=$ $\frac{s_{i j}}{e_{i j}}$. Otherwise, the optimal power allocation is determined by

$$
p_{i j}^{*}=\min \left(\left(\frac{x_{i j}^{*}}{\nu_{i}}-\frac{1}{e_{i j}}\right)^{+}, \frac{s_{i j}}{e_{i j}}\right),
$$

where the constant $\nu_{i}$ is chosen so that $\sum_{j \in \mathcal{N}} p_{i j}^{*}=P_{i}$. Note that it is possible that some subchannel is allocated to user $i$ but gets no power due to its (relatively) poor subchannel gain. The optimal value of $\nu_{i}$ can be found through a simple line search. However, it is possible to devise a finite-time algorithm (with a maximum of $2 n_{i}^{*}$ steps) to calculate the exact value of $v_{i}$ as in [3], [4] with the difference here being that this procedure needs to be executed for every user who is allocated a subchannel. Since $\sum n_{i}^{*}=N$, it follows that the total worst case computational complexity for this approach is $O(M N)$.

\section{Complexity Summary of Suboptimal Algorithms}

The worst case computational complexities of SOA1 and SOA2 are summarized in Table I.

\section{Simulation Results}

We report simulation results for the following four algorithms:

1) (Integer-Dual) integer subchannel allocation (with tie breaking) based on optimal algorithm as in Section IV and power control as in Section V-C, inspecting up to 128 ways of breaking the ties with an integer allocation and selecting the allocation among these with the largest weighted sum rate (before reallocating the power); 


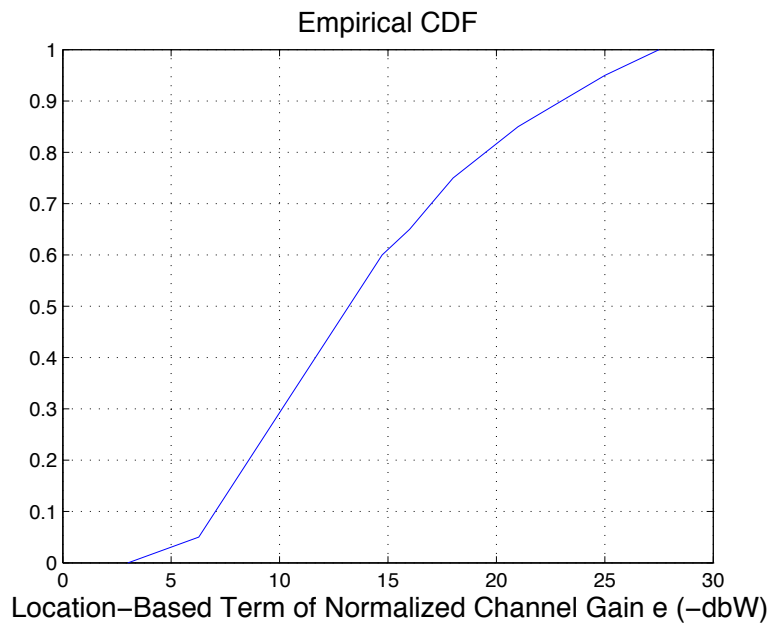

Fig. 1. Empirical CDF of the location-based term in users' normalized channel condition $e_{i j}$ 's for all user $i$ and subchannel $j$

2) (SOA1) all four versions of subchannel allocation procedure as in Section V-A, and power control as in Section $\mathrm{V}-\mathrm{C}$;

3) (SOA2) subchannel allocation as in Section V-B (with up to 10 iterations) and power control as in Section V-C;

4) (Base-line) each subchannel $j$ is allocated to the user $i$ with the highest $e_{i j}$, without considering the weights $w_{i}$ 's and the power constraints, while each user's power is still allocated as in Section V-C.

We report simulation results for the following four algorithms: 1) (Integer-Dual) integer subchannel allocation (with tie breaking) based on optimal algorithm as in Section IV and power control as in Section V-C, inspecting up to 128 ways of breaking the ties with an integer allocation and selecting the allocation among these with the largest weighted sum rate (before reallocating the power); 2) (SOA1) all four versions of subchannel allocation procedure as in Section V-A, and power control as in Section V-C; 3) (SOA2) subchannel allocation as in Section V-B (with up to 10 iterations) and power control as in Section V-C; and 4) (Base-line) each subchannel $j$ is allocated to the user $i$ with the highest $e_{i j}$, without considering the weights $w_{i}$ 's and the power constraints, while each user's power is still allocated as in Section V-C.

We consider a system bandwidth of $5 \mathrm{MHz}$ consisting of 512 OFDM tones, grouped into 64 subchannels ( 8 adjacent tones per subchannel, i.e., corresponding to the "Band AMC mode" of $802.16 \mathrm{~d} / \mathrm{e}$.). Each user's subchannel gains are the product of a constant location-based term, picked using an empirically obtained distribution, and a fast fading term, generated using a block-fading model and a standard mobile delay-spread model with a delay spread of $10 \mu \mathrm{sec}$. The fast-fading component for each multi-path component is held fixed for $2 \mathrm{msec}$ and an independent value is generated for the next block, which corresponds to a $250 \mathrm{~Hz}$ Doppler. The empirical cumulative distribution function (CDF) of the location-based term of users' location-based term of the $e_{i j}$ 's is given in Fig. 1, and the empirical $\mathrm{CDF}$ of the net $e_{i j}$ 's (i.e., including the fastfading component) is given in Fig. 2. Since the units of $e_{i j}$ are in $1 /$ watt, the $\mathrm{x}$-axis in both figures is measured in $-\mathrm{dBW}$.

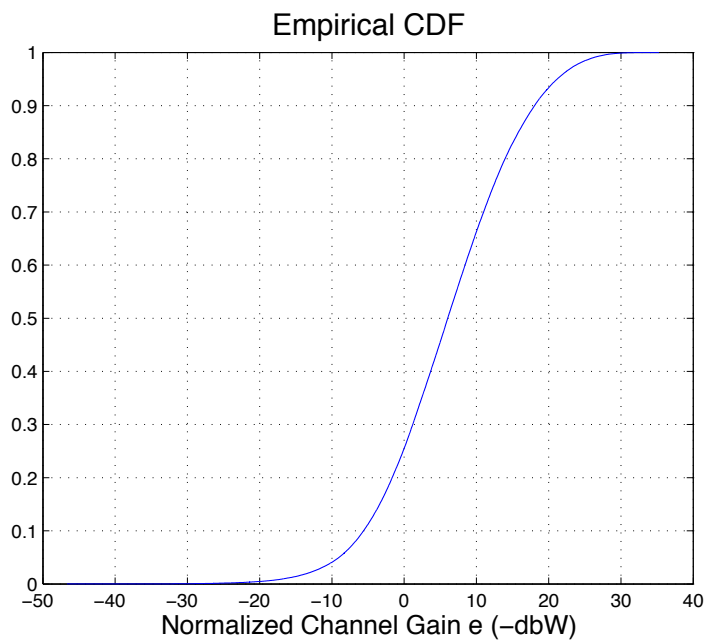

Fig. 2. Empirical CDF of users' normalized channel condition $e_{i j}$ 's for all user $i$ and subchannel $j$

The symbol duration is $100 \mu \mathrm{sec}$ with a cyclic prefix of $10 \mu \mathrm{sec}$, which roughly corresponds to 20 OFDM symbols per fading block (i.e., $2 m \mathrm{sec}$ ). This is one of the allowed configurations in the IEEE 802.16 standards [14]. Resource allocation (solving Problem UL) is done once per fading block. All the results are averaged over the last 1000 fading blocks. For the sake of illustration and to keep things simple, we assume that all users are infinitely back-logged, $d_{i}=0$, and have the same isoelastic utility function $u_{i}\left(W_{i, t}\right)=\left(W_{i, t}\right)^{\alpha} / \alpha$, where $W_{i, t}$ is the long term average throughput of user $i$ up to time $t$. All users also have the same maximum power constraint of $P_{i}=2 \mathrm{~W}$. We calculate the achievable rate of user $i$ on subchannel $j$ as

$$
r_{i j}=B x_{i j} \log \left(1+\frac{p_{i j} e_{i j}}{x_{i j}}\right),
$$

where $B$ is the subchannel bandwidth.

In all tables, the "Utility" column denotes $\sum_{i} U_{i}\left(W_{i, t}\right)$ (in scientific notations when appropriate), where $t$ is the end of simulation time. The "Log U" column denotes the logarithmic utility function, $\sum_{i} \log \left(W_{i, t}\right)$, which provides an alternate characterization of fairness among users. The "Rate" column denotes the total rate achieved by users in Mbps. The "User \#" column denotes the average number of users who receive positive rates within one scheduling interval. The "Opt. Ratio" for the Integer-Dual algorithm denotes the ratio between the objective value of Problem UL-Int achieved with the algorithm and the maximum objective value of Problem UL averaged over all scheduling instances. This gives us an idea of how good the performance of the Integer-Dual algorithm is. We emphasize that this value is based on the average performance of solving problem UL in each scheduling instance; this does not translate directly into a bound on the average utility under a optimal scheduling rule and under the given policy as the trajectory of the scheduling weights will be different under the two policies. Since other suboptimal algorithms do not find the optimal dual value of Problem UL at each scheduling instance, the "Optimality Ratio" does not apply to them.

Table II shows results for all the algorithms (summed over all users) when scheduling decisions are made every 20 OFDM symbols (i.e., a fading block of $2 \mathrm{msec}$ ). The utility parameter 
TABLE II

ALGORITHM PERFORMANCE FOR SCHEDULING EVERY 20 OFDM SYMBOLS, $\alpha=0.5$

\begin{tabular}{c|c|c|c|c|c|c}
\hline \hline \multicolumn{2}{c|}{ Algorithms } & Utility & Log U & Rate & User \# & Opt. Ratio \\
\hline \hline \multicolumn{2}{c|}{ Integer-Dual } & 53922 & 514.0 & 21.56 & 37.5 & 0.9412 \\
\hline \multirow{3}{*}{ SOA 1 } & 4A 5A & 52494 & 510.7 & 22.86 & 34.6 & N/A \\
\cline { 2 - 7 } & 4A 5B & 51697 & 509.2 & 20.22 & 28.1 & N/A \\
\cline { 2 - 7 } & 4B 5A & 54165 & 513.3 & 22.25 & 35.0 & N/A \\
\cline { 2 - 7 } & 4B 5B & 53156 & 511.4 & 21.43 & 28.6 & N/A \\
\hline \multicolumn{2}{c|}{ SOA 2 } & 54316 & 513.6 & 22.33 & 35.1 & N/A \\
\hline \multicolumn{2}{c|}{ Base Line } & 21406 & -1960.5 & 16.13 & 2.66 & N/A \\
\hline \hline
\end{tabular}

TABLE III

ALGORITHM PERFORMANCE FOR SCHEDULING EVERY 20 OFDM SYMBOLS, $\alpha=0$

\begin{tabular}{|c|c|c|c|c|c|c|}
\hline \multicolumn{2}{|c|}{ Algorithm } & Utility & $\overline{\log \mathrm{U}}$ & Rate & $\overline{\text { User \# }}$ & Opt. Ratio \\
\hline \multicolumn{2}{|c|}{ Integer-Dual } & $\overline{515}$ & $\overline{515}$ & 19.80 & $\overline{39.0}$ & 0.9715 \\
\hline \multirow{4}{*}{ SOA 1} & $4 \mathrm{~A} \quad 5 \mathrm{~A}$ & 511 & 511 & 18.47 & 36.9 & $\mathrm{~N} / \mathrm{A}$ \\
\hline & $4 \mathrm{~A} \quad 5 \mathrm{~B}$ & 509 & 509 & 21.56 & 28.6 & N/A \\
\hline & 4B $5 \mathrm{~A}$ & 515 & 515 & 20.04 & 37.4 & $\mathrm{~N} / \mathrm{A}$ \\
\hline & 4B $5 \mathrm{~B}$ & 512 & 512 & 17.80 & 29.3 & N/A \\
\hline \multicolumn{2}{|c|}{ SOA 2} & 515 & 515 & 19.91 & 37.4 & N/A \\
\hline \multicolumn{2}{|c|}{ Base Line } & -1961 & -1961 & 16.13 & 2.66 & N/A \\
\hline
\end{tabular}

is $\alpha=0.5$. In Table II, SOA1 (with 4B \& 5A) and SOA2 achieve the best performance in terms of total utility. Their performance is even better than the Integer-Dual approach, which was obtained based on the optimal value of the relaxed problem. This is likely because only 128 ways to break ties are considered, which is typically not sufficient. Since the IntegerDual algorithm achieves an optimality ratio of 0.9412 , this suggests that SOA1 and SOA2 achieve very close to optimal performance as well. The base-line algorithm always has poor performance.

Tables III and IV give the results of the algorithms when the utility parameter $\alpha$ is equal to 0 (proportional fair allocation) and 1 (maximum rate allocation), respectively. It is clear that there is a trade-off between fairness (measured by $\log \mathrm{U}$ ) and efficiency (measured by total rate). The value of $\alpha=0$ gives a throughput allocation that is the fairest and the least efficient, while $\alpha=1$ is the most efficient and least fair. Once again we note that all of the heuristics have good performance with SOA1 (with 4B \& 5A) and SOA2 achieving the best performances in terms of total utility.

In each case simulated, all of the SOA's have good performance with SOA1 (with 4B \& 5A) and SOA2 consistently achieving the best performance in terms of total utility. From the analysis in Section V-D, we note that these have slightly higher complexity than some of the other SOA's. Hence if lower complexity is desired, this can be provided with only a slight loss in performance. We also note that in each of the algorithms (except the base line one) a large number of users are scheduled in each time-slot, which may lead to a high signaling overhead. This can be addressed by adding a penalty term to our objective which increases with the number of users scheduled.

\section{CONCLUSIONS}

We presented an optimization-based formulation for scheduling and resource allocation in the uplink OFDM access
TABLE IV

ALGORITHM PERFORMANCE FOR SCHEDULING EVERY 20 OFDM SYMBOLS, $\alpha=1$

\begin{tabular}{c|c|c|c|c|c|c}
\hline \hline \multicolumn{2}{c|}{ Algorithm } & Utility & Log U & Rate & User \# & Opt. Ratio \\
\hline \hline \multicolumn{2}{c|}{ Integer-Dual } & $23.24 \mathrm{e} 6$ & 472.56 & 23.37 & 20.43 & 0.82541 \\
\hline \multirow{3}{*}{ SOA 1 } & 4A 5A & $23.19 \mathrm{e} 6$ & 448.99 & 22.28 & 22.6 & N/A \\
\cline { 2 - 7 } & 4A 5B & $23.11 \mathrm{e} 6$ & -136.03 & 23.20 & 15.6 & N/A \\
\cline { 2 - 7 } & 4B 5A & $24.31 \mathrm{e} 6$ & 444.02 & 24.42 & 32.7 & N/A \\
\cline { 2 - 7 } & 4B 5B & $23.95 \mathrm{e} 6$ & -195.10 & 24.05 & 15.6 & N/A \\
\hline \multicolumn{2}{c|}{ SOA 2 } & $24.46 \mathrm{e} 6$ & 372.95 & 24.57 & 21.8 & N/A \\
\hline \multicolumn{2}{c|}{ Base Line } & $16.08 \mathrm{e} 6$ & -1961 & 16.13 & 2.66 & N/A \\
\hline \hline
\end{tabular}

network. Compared to the downlink, we showed that the uplink was computationally more challenging due in part to the per-user power constraints. A (high complexity) optimal algorithm was given as well as a family of low complexity heuristics. The heuristics were shown to have good performance via simulations for a range of different user utilities and scheduling time-scales. Two algorithms from this family consistently achieved the best performance, but had a slightly higher complexity than some of the other algorithms, enabling complexity to be traded off with performance within this family of algorithms.

Finally, we want to mention that although we focus our discussions on the uplink transmissions in a single OFDM cell, the proposed algorithms can also be applied to downlink transmissions in a multi-cell OFDM system. A discussion of a more general solution framework which encompasses such cases is provided in [32].

\section{REFERENCES}

[1] R. Agrawal, A. Bedekar, R. La, V. Subramanian, "A Class and ChannelCondition based Weighted Proportionally Fair Scheduler," in Proc. ITC, Salvador, Brazil, Dec. 2001.

[2] R. Agrawal and V. Subramanian, "Optimality of Certain Channel Aware Scheduling Policies," in Proc. Allerton Conference, Oct. 2002.

[3] R. Agrawal, V. Subramanian and R. Berry, "Joint Scheduling and Resource Allocation in CDMA Systems," in Proc. of WiOpt '04, Cambridge, UK, March 2004.

[4] J. Huang, V. G. Subramanian, R. Agrawal and R. Berry, "Downlink scheduling and resource allocation for OFDM systems," IEEE Trans. on Wireless, to appear.

[5] L. Tassiulas and A. Ephremides, "Dynamic server allocation to parallel queue with randomly varying connectivity", IEEE Trans. IT, vol. 39, pp. 466-478, March 1993.

[6] R. Leelahakriengkrai and R. Agrawal, "Scheduling in Multimedia Wireless Networks," in Proc. ITC, Salvador, Brazil, pp. 556-564, Dec. 2001.

[7] M. Andrews, K. K. Kumaran, K. Ramanan, A. L. Stolyar, R. Vijayakumar, and P. Whiting, "Scheduling in a queueing system with asynchronously varying service rates," Probability in Engineering and Informational Sciences, vol. 18, No. 2, pp. 191-217, 2004.

[8] A. Jalali, R. Padovani, R. Pankaj, "Data throughput of CDMA-HDR a high efficiency - high data rate personal communication wireless system.," in Proc. IEEE VTC, Spring 2000, vol. 3, pp. 1854-1858.

[9] A. L. Stolyar, "Maximizing Queueing Network Utility subject to Stability: Greedy Primal-Dual Algorithm," Queueing Systems, vol. 50, pp. 401-457, 2005.

[10] A. L. Stolyar, "On the asymptotic optimality of the gradient scheduling algorithm for multiuser throughput allocation," Operations Research, vol. 53, No. 1, pp. 12-25, 2005.

[11] M. Andrews, L. Qian, and A. Stolyar, "Optimal utility based multi-user throughput allocation subject to throughput constraints," in Proc. IEEE INFOCOM, March 2005, vol. 4, pp. 2415-2424.

[12] H. Kushner and P. Whiting, "Asymptotic properties of proportional-fair sharing algorithms," in Proc. Allerton Conference, Oct. 2002.

[13] J. Mo and J. Walrand, "Fair end-to-end window-based congestion control," IEEE/ACM Trans. Networking, vol. 8, Issue 5, pp. 556-567, Oct. 2000. 
[14] “IEEE 802.16e-2005 and IEEE Std 802.16-2004/Cor1-2005," 2005, Available: http://www. ieee802.org/16/.

[15] E. D. Nering and A. W. Tucker, Linear Programs and Related Problems. Academic Press Inc., 1993.

[16] H. Yin and H. Liu, "An efficient multiuser loading algorithm for OFDMbased broadband wireless systems," in Proc. IEEE Globecom, vol. 1, pp. 103-107, Dec. 2000.

[17] T. M. Cover and J. A. Thomas, "Elements of information theory," Second edition, Wiley-Interscience, Hoboken, NJ, 2006.

[18] S. Shenker, "Fundamental design issues for the future Internet," IEEE J. Select. Areas Commun., vol. 13, no. 7, pp. 1176-1188, 1995.

[19] FLASH-OFDM Technology, http://www.qualcomm.com/technology/ flash-ofdm

[20] D. Bertsekas, Nonlinear Programming, 2nd ed. Belmont, Massachusetts: Athena Scientific, 1999.

[21] Z. Han, Z. Ji, and K. Liu, "Fair Multiuser Channel Allocation for OFDMA Networks Using Nash Bargaining Solutions and Coalitions," IEEE Trans. Commun., vol. 53, no. 8, pp. 1366-1376, 2005.

[22] S. Pfletschinger, G. Muenz, and J. Speidel, "Efficient subcarrier allocation for multiple access in OFDM systems," in Proc. International OFDM-Workshop, 2002.

[23] Y. Ma, "Constrained Rate-Maximization Scheduling for Uplink OFDMA," in Proc. IEEE MILCOM, pp. 1-7, Oct. 2007.

[24] B. Da and C. Ko, "Dynamic subcarrier sharing algorithms for uplink OFDMA resource allocation," in Proc. 6th ICICS, pp. 1-5, 2007.

[25] K. Kim, Y. Han, and S. Kim, "Joint subcarrier and power allocation in uplink OFDMA systems," IEEE Commun. Lett., vol. 9, no. 6, pp. $526-528,2005$.

[26] C. Ng and C. Sung, "Low complexity subcarrier and power allocation for utility maximization in uplink OFDMA systems," IEEE Trans. Wireless Commun., vol. 7, no. 5 Part 1, pp. 1667-1675, 2008.

[27] L. Gao and S. Cui, "Efficient subcarrier, power, and rate allocation with fairness consideration for OFDMA uplink," IEEE Trans. Wireless Commun., vol. 7, no. 5 Part 1, pp. 1507-1511, 2008.

[28] K. Kwon, Y. Han, and S. Kim, "Efficient Subcarrier and Power Allocation Algorithm in OFDMA Uplink System," IEICE Trans. Commun., vol. 90, no. 2, pp. 368-371, 2007.

[29] "3GPP Long Term Evolution," 2008, Available: http://www.3gpp.org/ Highlights/LTE/LTE.htm .

[30] “3GPP2 Ultra Mobile Broadband," 2007, Available: http://www.3gpp2. org/ .

[31] W. Yu, R. Lui, and R. Cendrillon, "Dual optimization methods for multiuser orthogonal frequency division multiplex systems," in Proc. IEEE Globecom, vol. 1, 2004, pp. 225-229.

[32] J. Huang, V. G. Subramanian, R. Berry, and R. Agrawal, "Scheduling and Resource Allocation in OFDMA Wireless Systems," book chapter, submitted.

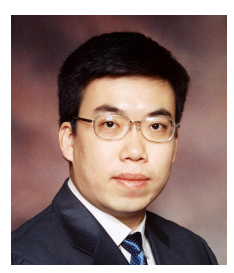

Jianwei Huang (S'01-M'06) is an Assistant Professor in Information Engg. Department at the Chinese University of Hong Kong. He received the M.S. and Ph.D. degrees in Electrical and Computer Engg. from Northwestern University in 2003 and 2005, respectively. From 2005 to 2007, He worked as a Postdoctoral Research Associate in the Department of Electrical Engg. at Princeton University. His main research interests lie in the area of modeling and performance analysis of communication networks, including cognitive radio networks, OFDM and CDMA systems, wireless medium access control, multimedia communications, network economics, and applications of optimization theory and game theory. Dr. Huang is an Associate Editor of Journal of Computer \& Electrical Engg., the Lead Guest Editor of the IEEE Journal of Selected Areas in Communications special issue on "Game Theory in Communication Systems", the Lead Guest Editor of the Journal of Advances in Multimedia special issue on "Collaboration and Optimization in Multimedia Communications", and a Guest Editor of the Journal of Advances in Multimedia special issue on "Cross-layer Optimized Wireless Multimedia Communications", the Technical Program Committee Co-Chair of the International Conference on Game Theory for Networks (GameNets'09).

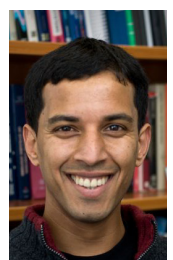

Vijay G. Subramanian (M'01) received his Ph.D. degree in Electrical Engg. from the University of Illinois at Urbana-Champaign, in 1999. From 1999 to 2006, he was with the Networks Business, Motorola, Arlington Heights, IL, USA. Since May 2006 he is a Research Fellow at the Hamilton Institute, NUIM, Ireland. His research interests include information theory, communication networks, queueing theory, and applied probability and stochastic processes.

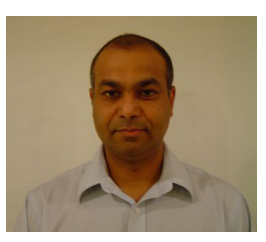

Rajeev Agrawal is a Fellow of the Technical Staff at Motorola where his responsibilities include the architecture, design and optimization of Motorola's next generation wireless systems. Prior to joining Motorola in 1999, Rajeev was Professor of Electrical and Computer Engg. and Computer Science departments at the University of Wisconsin - Madison. He also spent a sabbatical year at IBM TJ Watson Research, British Telecom Labs, and INRIA-Sophia Antipolis. Rajeev received his M.S. (1987) and Ph.D. (1988) degrees in Electrical Engg.-systems from the University of Michigan, Ann Arbor and his B.Tech. (1985) degree in Electrical Engg. from the Indian Institute of Technology, Kanpur.

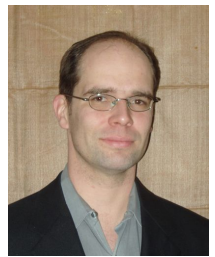

Randall A. Berry (S'93-M'00) received the M.S. and $\mathrm{PhD}$ degrees in Electrical Engg. and Computer Science from the Massachusetts Institute of Technology in 1996 and 2000, respectively. In September 2000 , he joined the faculty of Northwestern University, where he is currently an Associate Professor of Electrical Engg. and Computer Science. Dr. Berry is the recipient of a 2003 NSF CAREER award. He is currently serving on the editorial boards of the IEEE Transactions on Information Theory and the IEEE Transactions on Wireless Communications. 